\title{
Farmacoterapia no traumatismo craniano. Onde estamos? Para onde vamos? Porém, quando vamos?
}

\section{Pharmacotherapy in Traumatic Brain Injury. Where we are? Where we are going? However, when?}

\author{
${ }^{1}$ Acadêmica; Medicina da Universidade Estadual de Ponta Grossa \\ (UEPG), Ponta Grossa, PR, Brasil \\ 2 Neurocirurgião; Professor Adjunto de Neurocirurgia da UEPG, Ponta \\ Grossa, PR, Brasil \\ ${ }^{3}$ Neurocirurgião; Professor Assistente da Universidade de São Paulo \\ (USP), São Paulo, SP, Brasil
}

Mariana Schumacher Welling ${ }^{1}$ Leonardo C. Welling ${ }^{2}$ Eberval Gadelha Figueiredo ${ }^{3}$

Arq Bras Neurocir 2015;34:208-214.

\section{Resumo \\ Palavras-Chave \\ - Traumatismo Craniano \\ - Reabilitação \\ - Farmacoterapia}

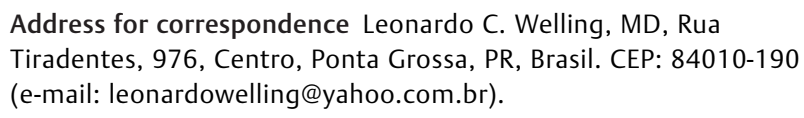

\begin{abstract}
Keywords

- Traumatic Brain Injury

- Rehabilitation

- Pharmacotherapy

Traumatic brain injury (TBI) is an increasingly prevalent and complex challenge for society. Primary injuries are defined as those that occur at the moment of impact. Secondary injuries are those that occur after the initial injury as a consequence of physiologic / pathologic response to injury. This triggers a cascade of pathophysiological events that can extend over a long period of time. Whereas the primary injuries are considered irreversible, secondary injuries are potentially preventable with efficient triage and stabilization, management of parameters such as brain oxygenation, intracranial pressure, and cerebral perfusion pressure. Indeed, TBI should be thought of not as a static event, but rather a progressive injury with varying therapeutic windows. In this context the development of pharmacological treatment of patients
\end{abstract}

O traumatismo cranioencefálico (TCE) apresenta aumento de sua prevalência e é um desafio para a sociedade atual. Lesões primárias são aquelas que ocorrem no momento do impacto. Lesões secundárias são as que ocorrem após a lesão inicial como uma resposta fisiológica/patológica. Isso causa uma cascata de eventos que pode durar um longo período. Enquanto as lesões primárias são irreverssíveis, as secundárias são potencialmente evitáveis se forem submetidas a triagem e estabilização adequadas, assim como ao correto manejo da oxigenação cerebral e controle da hipertensão intracraniana e pressão de perfusão cerebral. Devemos considerar o traumatismo craniano como um evento dinâmico com inúmeras janelas terapêuticas possíveis. Nesse contexto o desenvolvimento de agentes farmacológicos no tratamento do TCE é urgente. Na presente revisão os autores descrevem os principais fármacos que estão sendo estudados nos pacientes com traumatismo craniano. Concluímos que, apesar de investimentos substanciais em estudos de fase I e II, ainda há muitas lacunas no conhecimento, portanto esforços são necessários para que haja uma translação mais rápida para estudos de fase III.

received

July 7, 2014

accepted

June 12, 2015
DOI http://dx.doi.org/ $10.1055 / \mathrm{s}-0035-1559890$ ISSN 0103-5355.
Copyright $\odot 2015$ by Thieme Publicações License terms Ltda, Rio de Janeiro, Brazil

c. $9 \triangleq \$$ 
with traumatic brain injury is urgent. In this review the authors describe the main drugs that are being studied in TBI patients. We conclude, despite substantial and ongoing investments in both phase I and II studies, there remain significant gaps in knowledge and faster translation to phase III clinical studies is mandatory.

\section{Introdução}

A incidência de trauma cranioencefálico (TCE) aumenta em todo o mundo, seja por acidentes automobilísticos, ou pelo aumento da população idosa, com o consequente crescimento de acidentes domésticos (quedas) e uso de anticoagulantes. Após o trauma, as intervenções aplicadas para minimizar os eventos inflamatórios secundários baseiamse em sedação, uso de manitol, solução salina hipertônica, hiperventilação e medidas cirúrgicas. Com o avanço das medidas de monitoração neurológica multimodal e o conhecimento fisiopatológico dos eventos inflamatórios secundários no sistema nervoso central lesionado, novas medidas farmacológicas são estudadas, sejam em modelos animais ou em ensaios clínicos de fase I ou II. Na presente revisão, propomos descrever os principais medicamentos estudados e seus impactos na neurotraumatologia atual.

\section{Amantadina}

A amantadina é uma amina tricíclica criada para profilaxia e tratamento da gripe influenza A, entretanto foi consagrada no tratamento da doença de Parkinson. Apesar do mecanismo antiparkinsoniano não estar esclarecido, sabe-se que a droga aumenta as concentrações extracelulares de dopamina, seja pelo bloqueio de recaptação ou pelo aumento na sua síntese. ${ }^{1}$ Além, ao aumentar a afinidade pelo seu receptor, amplifica os efeitos da dopamina nos circuitos póssinápticos. $^{2}$

Em modelos animais de traumatismo craniano o uso da amantadina foi pouco estudado. De acordo com Dixon et al., nos ratos que usaram a droga desde o primeiro dia do impacto até o $18^{\circ}$ dia, observou-se melhora modesta no labirinto aquático de Morris; entretanto, não houve melhora da função motora nem aumento da sobrevida dos neurônios hipocampais. $^{3}$

Dois ensaios clínicos randomizados demonstraram eficácia modesta em pacientes que apresentavam distúrbios de consciência após TCE, todavia o número pequeno da amostra e outras limitações metodológicas reduziram seu impacto. ${ }^{4,5}$

Recentemente, Whyte et al. conduziram um estudo observacional multicêntrico, que comparou a eficácia do placebo com a da amantadina nos pacientes em estado vegetativo persistente ou em estado de consciência mínima durante 4 a 16 semanas após o trauma. Por meio da análise de regressão multivariada, a amantadina foi associada a melhora na escala DRS (Disability Rating Scale) quando comparada ao uso de placebo. Este estudo demonstrou que determinadas intervenções farmacológicas podem interferir na recuperação de pacientes após o TCE e levou a novo estudo de fase III que demonstrou a eficácia do uso de amantadina em pacientes em estado vegetativo ou em estado de consciência mínima. Observou-se que o grupo submetido a tratamento com amantadina obteve melhor pontuação na esclara DRS quando comparado ao grupo placebo. ${ }^{6}$

Além do momento de iniciar-se a amantadina, alguns estudos ainda são necessários para observar quais são os pacientes mais beneficiados, a duração do tratamento, os marcadores para avaliação de eficácia da droga e a possível utilização desta em pacientes com traumatismo craniano leve.

\section{Inibidores da Acetilcolinesterase}

Ao bloquear a enzima que degrada a acetilcolina, os inibidores da acetilcolinesterase aumentam a concentração desta na fenda sináptica. Apesar de inicialmente aprovada para o tratamento da demência de Alzheimer em suas formas leves e moderadas, há muitos estudos no tratamento de transtornos cognitivos após traumatismo. ${ }^{7}$ Dados iniciais demonstraram a eficácia da fisostigmina; entretanto, o advento de inibidores da acetilcolinesterase com perfil mais seguro ocasionou o atual aumento do número de ensaios clínicos com a donepezila, rivastigmina e galantamina nos pacientes com sequelas cognitivas após traumatismo craniano grave. ${ }^{7-11}$

Há evidências de que o aumento dos níveis de acetilcolina implica melhora cognitiva nos pacientes vítimas de traumatismo craniano grave. ${ }^{12}$ Estudos sobre animais mostram efeitos positivos na fase aguda do trauma com redução da morte neuronal, preservação dos neurônios hipocampais (região CA1), menor ruptura da barreira hematoencefálica, redução do edema cerebral vasogênico e preservação da função neurológica. ${ }^{7,13}$ Nesses estudos a acetilcolina foi responsável pela neuroproteção, visto que os efeitos neuroprotetores foram antagonizados por bloqueadores dos receptores nicotínicos e/ou muscarínicos.

Apesar de inúmeras publicações com a donepezila na reabilitação neurológica após o trauma, há apenas dois ensaios clínicos randomizados com esse fármaco. Pelas falhas metodológicas e pelo pequeno número amostral, a eficácia da donepezila comparada ao tratamento convencional ainda não foi demonstrada. ${ }^{7}$

Para a rivastigmina há dois ensaios clínicos de fase II já completados que demonstraram a segurança do medicamento nos pacientes com traumatismo craniano moderado e grave. ${ }^{14,15}$ Esses mesmos dois trabalhos não demonstraram eficácia na avaliação neuropsicológica na $12^{\text {a }}$ semana após o tratamento. Atualmente, há um estudo de fase II que vai 
avaliar a segurança da rivastigmina transdérmica nesses pacientes. $^{16}$

Um quarto ensaio clínico - CREATE (Cognitive Remediation After Trauma Exposure) - recrutou pacientes para a comparação entre galantamina, metilfenidato e placebo no tratamento dos sintomas cognitivos após traumatismo craniano e transtorno de estresse pós-traumático, porém seus resultados ainda não foram publicados. ${ }^{17}$

\section{Huperzina A}

A huperzina A é um alcaloide natural extraído da planta chinesa huperzia serrata, utilizada para o tratamento da demência senil e doença de Alzheimer na China. Além disso, pode ser usada como anticonvulsivante, posto que se comporta como antagonista do N-metil-D-aspartato, sendo importante terapia coadjuvante em pacientes que sofreram um TCE grave. Nos Estados Unidos, é mais utilizada como fitoterápico no tratamento de distúrbios da memória. ${ }^{18}$

Estudos recentes sugerem que ela apresenta efeitos neuroprotetores por ativar o sistema colinérgico e por acelerar o metabolismo do precursor da proteína B-amiloide, diminuindo assim o estresse oxidativo mitocondrial causado pelo trauma. $^{18}$

0 estudo em modelo animal mais recente analisou o mecanismo de ação da huperzina através da modulação das vias amiloidogênicas e não amiloidogênicas. ${ }^{19}$ Existem atualmente poucos estudos que provam a eficácia da terapêutica com huperzina no transtorno cognitivo, sendo a maioria deles relacionados a melhora do déficit em pacientes com doença de Alzheimer. ${ }^{20}$

De acordo com Li et al., há seis ensaios clínicos que analisam a segurança e eficácia do fármaco quando utilizado após TCE. Alguns desfechos favoráveis foram vistos, como melhora da função cognitiva, estado clínico geral e desempenho funcional. ${ }^{21}$

Novos estudos são necessários para avaliar a eficácia desse fármaco. Atualmente, um novo estudo clínico está em andamento (NCT 01676311), comparando a huperzina com o placebo no aprendizado e memória dos pacientes que foram vítimas de traumatismo craniano grave. ${ }^{16}$

\section{Ciclosporina}

O principal mecanismo postulado para neuroproteção com ciclosporina é o bloqueio da permeabilidade na membrana mitocondrial após o trauma, o que mantém sua integridade e seu potencial de ação, com isso a produção de espécies reativas de oxigênio fica reduzida. ${ }^{22,23}$ Sua atividade imunossupressora exerce efeitos benéficos sobre a lesão axonal, o aprendizado e a memória. ${ }^{24}$

Há vários estudos pré-clínicos que demonstram desfechos histológicos benéficos em modelos animais de traumatismo craniano, inclusive no dano axonal e nos volumes contusionais, além de atuação sinérgica com a hipotermia. Apesar disso, estudos que avaliam o desfecho motor são apenas dois. $^{24,25}$
A despeito dos resultados benéficos, há outros estudos em ratos que não demonstraram redução da concentração de neuromarcadores inflamatórios e observaram que a ciclosporina não foi responsável pela melhora da resposta cognitiva. $^{26}$

Ainda há necessidade de definir os biomarcadores farmacológicos que serão utilizados para a avaliação da eficácia, bem como o início da administração e a dose efetiva do fármaco. Alguns autores sugerem a infusão terapêutica cerca de 15 minutos até 1 hora após o trauma, mas de acordo com Sullivan et al. pode haver benefício se for administrada nas 8 primeiras horas. ${ }^{27}$ Atualmente, há cinco estudos clínicos de fase II que têm por objetivo avaliar os efeitos dessas drogas. ${ }^{16}$

Empey et al. realizaram um estudo de escalonamento de dose focada em farmacocinética e amostras de plasma de trinta pacientes com TCE grave. As doses utilizadas foram de $0,625-2,5 \mathrm{mg} / \mathrm{kg}$, e a ciclosporina foi liberada mais rapidamente e teve um volume de distribuição maior nos pacientes com TCE do que na população controle. ${ }^{28}$

Mazzeo et al. demonstraram os efeitos imunológicos da ciclosporina em 59 pacientes com trauma grave, utilizando uma dose de $5 \mathrm{mg} / \mathrm{kg}$ em uma ou duas infusões nas primeiras 24 horas. O resultado neurológico, imunidade celular e taxa de infecção não diferiram entre os grupos ciclosporina e placebo. Um estudo posterior analisou efeitos metabólicos e hemodinâmicos da ciclosporina em cinquenta adultos com TCE grave usando uma dose de $5 \mathrm{mg} / \mathrm{kg}$ versus placebo. 0 seu uso foi associado a um significativo aumento da pressão arterial média (PAM) e da pressão de perfusão cerebral (PPC). No grupo tratamento com ciclosporina foi utilizado cateteres de microdiálise cerebral e evidenciou-se níveis intersticiais elevados de lactato e gliose, enquanto a concentração de glutamato e a relação lactato/piruvato foram reduzidas em relação ao grupo placebo. ${ }^{29}$

Hatton et al estudaram quarenta adultos com TCE grave, 32 tratados com ciclosporina e $8 \mathrm{com}$ placebo. Não foram observadas diferenças na mortalidade ou na incidência de complicações entre os grupos, apesar de um efeito positivo em 6 meses, na pontuação de acordo com a Glasgow Outcome Scale - nos pacientes que usaram ciclosporina. ${ }^{30}$

De acordo com Aminmansour et al, pacientes que utilizaram ciclosporina após lesão axonal difusa não apresentaram melhora no estado de consciência e no padrão comportamental quando comparados aos pacientes que utilizaram placebo. ${ }^{31}$

Apesar dos benefícios histológicos e imunológicos, a permeabilidade reduzida da barreira hematoencefálica a ciclosporina, a neurotoxicidade ocasionada por seu uso crônico e os resultados controversos podem limitar sua aplicabilidade clínica. ${ }^{16}$

\section{Glibenclamida}

A glibenclamida, droga popularmente utilizada para o tratamento do diabetes, é uma sulfanilureia que se liga aos receptores SUR1 e com isso bloqueia os canais potássio-ATP dependentes. $\mathrm{O}$ interesse por esse fármaco advém do 
conhecimento que as proteínas SUR1 estão expressadas em células neuronais após qualquer tipo de lesão. ${ }^{32}$

Há inúmeros estudos pré-clínicos utilizando modelos de trauma, hemorragia subaracnoide e isquemia sobre os efeitos da glibenclamida no sistema nervoso central. Indicam que seu uso reduz inflamação, hemorragia e edema vasogênico. ${ }^{16}$ Dados recentes demonstram que a droga, quando iniciada minutos após a lesão, reduz a gravidade de hemorragias secundárias e as lesões hipocampais. ${ }^{33}$ Outro ensaio clínico de fase II evidenciou melhora do edema e redução da necessidade de osmoterapia em pacientes que usaram a sulfonilureia quando comparados a placebo. ${ }^{32}$

Atualmente há um outro estudo de fase II que tem como objetivo mensurar com ressonância magnética a formação de edema e hemorragia nos pacientes com TCE grave tratados por 72 horas após a lesão primária. ${ }^{16}$

\section{Hormônio de Crescimento}

O hormônio do crescimento (GH) é um aminoácido de cadeia única, sintetizado, armazenado e secretado pelas células somatotróficas da glândula hipófise. Sua liberação é pulsátil e coordenada por estímulos hipotalâmicos. Há vários receptores para esse hormônio nas mais distintas localizações do parênquima encefálico, o que sugere papel funcional do $\mathrm{GH}$ em diversas atividades cerebrais. Cerca de $20 \%$ dos pacientes vítimas de traumatismo craniano cursam com insuficiência na produção de $\mathrm{GH}$, e essa condição pode decorrer de lesão direta da hipófise ou lesão indireta no hipotálamo. ${ }^{34}$

Modelos experimentais em ratos evidenciam melhora na função motora, retenção de memória e aprendizado. ${ }^{16,34,35}$ Estudos recentes evidenciam que seu uso em pacientes após TCE pode ter efeitos neuroprotetores e neuroregenerativos. ${ }^{35}$ O GH também aumenta o fator de crescimento derivado da insulina (IGF-1), que ao agir nas células endoteliais progenitoras auxilia na reparação da parede vascular lesionada. ${ }^{35}$

Há estudos prospectivos que demonstraram melhora cognitiva em pacientes que utilizaram GH recombinante humano. ${ }^{36} \mathrm{O}$ tempo médio de tratamento foi de 8 a 12 meses, e o tempo médio decorrido do traumatismo craniano foi de 11 anos. Observa-se que em nenhum dos estudos os efeitos adversos comprometeram a segurança de seu uso. Entretanto, estudos anteriores realizados na Europa, com doses suprafisiológicas do hormônio do crescimento, foram interrompidos em virtude do aumento da mortalidade. ${ }^{37}$

Apesar das evidências atuais de eficácia do uso de GH no tratamento agudo e crônico do paciente com TCE associado a insuficiência de GH documentada, a inexistência de estudos de fase III não permite o dimensionamento dos resultados. Além disso, não há dados que assegurem a dosagem efetiva para os pacientes durante a fase aguda do trauma. ${ }^{16}$

\section{Lítio}

Amplamente utilizado no tratamento de transtorno bipolar, exerce também função de neuroproteção por reduzir a excitotoxicidade, a isquemia e a apoptose. Age também atenuando a expressão de diversas citocinas pró-inflamató- rias - como BACE-1, gsk-3ß, COX2-e preserva a integridade da barreira hematoencefálica. ${ }^{38}$

Em modelos animais, Yu et al. administraram lítio 15 minutos antes da injúria cerebral e por 3 semanas após o evento. Houve melhora da orientação espacial e do comportamento medida por escalas específicas. Esse mesmo estudo também demonstrou que o volume da lesão foi reduzido e que o uso da droga deve ser feito em até 3 horas após a lesão. ${ }^{38}$ Corroborando os achados desse, Zhu et al utilizaram lítio em roedores antes e depois do TCE e encontraram alguns resutados benéficos como a diminuição do edema e a expressão das citocinas pró-inflamatórias. ${ }^{39}$

Na esfera clínica, há seis pequenas séries em que o lítio foi utilizado em pacientes após traumatismo craniano. Os principais objetivos foram o controle da agitação e do comportamento agressivo. As doses variaram entre 600 e $1.200 \mathrm{mg} / \mathrm{dia}$ e os desfechos foram variados, com melhora em alguns e piora em outros casos. Em nenhum desses trabalhos utilizouse medidas objetivas como neuroimagem ou análise histológica após morte. ${ }^{16}$

\section{Metilfenidato e Atomoxetina}

O metilfenidato e a atomoxetina são responsáveis pelo aumento de dopamina e noradrenalina na fenda sináptica e também da noradrenalina. ${ }^{40}$ Ambos são utilizados para o tratamento de déficit de atenção e hiperatividade. Alguns estudos pré-clínicos têm por finalidade demonstrar a eficácia dessas medidas terapêuticas nos pacientes após oTCE. Os resultados encontrados apontam para melhora da neurotransmissão dopaminérgica no estriado e melhora na aprendizagem e desempenho motor. ${ }^{41}$

Existem cerca de quinze estudos clínicos que utilizaram metilfenidato nos pacientes vitimas de TCE moderado e severo na sua fase crônica e subaguda. Os estudos foram, em geral, pequenos, com menos de quarenta casos por estudo e com variação da duração de tratamento desde uma dose em 1 dia até durante 30 dias. ${ }^{16} \mathrm{O}$ principal objetivo era avaliar a segurança do metilfenidato nos pacientes vítimas de traumatismo craniano. ${ }^{16}$

Nos estudos que avaliaram os resultados neuropsicológicos, não foi observada melhora na atenção nem na excitação. Sete ensaios clínicos avaliaram o processamento e velocidade de reação, mas apenas um demonstrou resultado positivo, tendo dois estudos demonstrado achados negativos em relação ao uso do medicamento. ${ }^{16}$

Dentre os oito ensaios clínicos randomizados que avaliaram o comportamento após o uso de metilfenidato, um deles relatou melhora na escala de depressão de Hamilton, mas não na escala de Beck. ${ }^{16}$

Segundo Moein et al, o tratamento com metilfenidato na fase aguda do trauma ( $<48$ horas) diminui significativamente o tempo de internação na unidade de terapia intensiva. $^{42}$

Embora os estudos clínicos do uso do metilfenidato sejam bastante heterogêneos em sua metodologia, população, duração do tratamento e também por serem amostras pequenas, uma análise de todos eles demonstra que essa droga pode ter um 
papel útil na terapêutica do TCE. Para melhor compreensão do seu potencial terapêutico, é necessário que sejam feitos estudos clínicos com populações maiores, além de mais estudos préclínicos em modelos animais, com ferramentas que evidenciem melhor os resultados, como exame de imagem.

\section{Minociclina}

É um antibiótico que pertence ao grupo das tetraciclinas e, por ser lípide, atravessa facilmente a barreira hematoencefálica. Há modelos que demonstram a eficácia da droga na isquemia cerebral, doença de Huntington, esclerose múltipla e doença de Alzheimer. ${ }^{43}$ Estudos laboratoriais demonstram que possui atividade anti-inflamatória, antiapoptótica e antioxidante muito maior do que o necessário para a atividade antimicrobiana, e nesse contexto passou a ser estudada na neurotraumatologia. ${ }^{16}$

Estudos pré-clínicos, em modelos animais, demonstram que o uso de minociclina, quando administrada nos primeiro 45 minutos após o trauma, foi responsável por menor volume de lesão, além de preservar o conteúdo de mielina. ${ }^{44} \mathrm{Se}-$ gundo Kovesdi et al., a minociclina diminui os níveis dos biomarcadores de lesão neuronal e glial quando administrada nas primeiras 4 horas após a lesão. ${ }^{45}$

\section{N-Acetilcisteína}

A N-acetilcisteína (NAC) é um fármaco utilizado primariamente como agente mucolítico, sendo prescrito para as mais diversas condições pulmonares. Também é a droga de escolha para o tratamento de intoxicação por paracetamol. A administração pode ser via oral, intravenosa ou por aerossol. Após a absorção, é metabolizada no fígado, onde sofre um processo de desacetilação, formando a cisteína. ${ }^{46}$ Tanto a NAC quanto a cisteína são potentes agentes antioxidantes, porém a cisteína desempenha função importante no aumento dos níveis de glutationa e glutamato no cérebro. ${ }^{47}$

Em modelos animais de injúria cerebral, exerce uma forte ação antioxidante ao observar-se os marcadores de lesão oxidativa. Ao seu uso também é atribuída a redução no volume de lesão. ${ }^{48}$ Nos estudos em que ela foi utilizada a partir da primeira hora após a lesão, não se observou redução do edema nem do volume da lesão cerebral. ${ }^{48}$ Em um estudo randomizado realizado por Hoffer et al. com 81 pacientes que sofreram algum grau de concussão cerebral, observou-se melhora dos sintomas nos pacientes que utilizaram NAC por 7 dias quando comparados ao grupo placebo. ${ }^{49}$

Apesar de existirem atualmente vários ensaios clínicos que utilizam NAC para distúrbios neurológicos e psicológicos devido a suas atividades antioxidantes, anti-inflamatórias e neuromoduladoras, tendo poucos efeitos colaterais indesejáveis, ainda não há evidências suficientes de sua eficácia e do tempo de tratamento. ${ }^{16}$

\section{Progesterona}

A progesterona é um hormônio esteroide que, além de ser sintetizado nos órgãos reprodutivos e nas glândulas suprar- renais, é produzido no cérebro, onde as concentrações teciduais são 5 vezes maior que a concentração sérica.

Estudos em modelos animais apontam para possíveis benefícios na utilização da progesterona na lesão cerebral, como neuroproteção, melhora da capacidade funcional e comportamental, redução do edema cerebral, da apoptose, da concentração de citocinas inflamatórias, além do aumento da mielinização e da neurogênese. ${ }^{50}$

Adicionalmente, a progesterona quando administrada via oral aumenta a concentração de alopregnanolona, um neuroesteroide proveniente da metabolização da progesterona, que modula os receptores GABAa e com isso exerce efeitos neuroprotetores significativos. ${ }^{51}$ Dados pré-clínicos sugerem que, quando administrada concomitantemente à vitamina $\mathrm{D}$, há potencialização dos efeitos neuroprotetores. ${ }^{52}$

Estudos pré-clínicos demonstram que a progesterona diminui o edema cerebral após o TCE, reduz a apoptose e a concentração de citocinas pró-inflamatórias como IL-6, IL-1b e o fator de necrose tumoral. Além disso, protege contra a peroxidação lipídica ao aumentar a atividade da superóxido dismutase e os níveis dos fatores de neurotróficos, inibe a sinalização do cálcio neuronal, diminui a pressão intracraniana, modula a expressão das aquaporinas, diminui a disfunção mitocondrial, reduz o acúmulo astrocitário e a lesão axonal após a lesão. ${ }^{53}$

Outro estudo pré-clínico associa seu uso a melhora funcional e comportamental, tanto na aprendizagem quanto na memória, bem como na atividade de locomoção. ${ }^{54}$ Apesar dos resultados favoráveis observados nos ensaios pré-clínicos, ainda não há ensaios clínicos concluídos que demonstrem o benefício do fármaco nos humanos.

\section{Estatinas}

As estatinas são usadas principalmente para reduzir os níveis de colesterol sérico, entretanto, seus efeitos pleiotrópicos são responsáveis pela repercussão que essa classe de medicamentos exerce nas áreas relacionadas a patologias do sistema nervoso central. Existem evidências em modelos animais que demonstram efeitos benéficos sobre o edema cerebral, integridade da barreira hematoencefálica, fluxo sanguíneo cerebral, neuroinflamação e lesão axonal. ${ }^{55}$

Segundo Wang et al, a atorvastatina seria superior a sinvastatina ao relacionar-se a menor índice de morte neuronal e ter maior meia-vida. ${ }^{56}$ Entretanto, Lu et al. referem que ratos que utilizaram sinvastatina em comparação com a atorvastatina apresentam menor morte de células hipocampais. ${ }^{57}$ Serra et al. compararam os efeitos de nove estatinas distintas e descrevem que o melhor efeito ocorre com o uso da sinvastatina por ela apresentar maior penetração na barreira hematoencefálica e maior proteção contra a neurodegeneração das proteínas tau. ${ }^{58} \mathrm{O}$ quanto isso será benéfíco para os pacientes com traumatismo craniano ainda é incerto, mas a lipossolubilidade da sinvastatina não pode ser descartada quando o assunto é maior penetração no sistema nervoso central. A via de administração na maioria dos estudos é oral, e a janela terapêutica é favorável em até 24 horas após o trauma. A duração do tratamento é de 7 a 14 dias. $^{16}$ 
Atualmente está em andamento um estudo de fase II de segurança e eficácia da atorvastatina em traumatismo craniano leve em que o desfecho primário será a pontuação obtida no questionário de Rivermead no terceiro mês após a lesão. ${ }^{16}$ Para o tratamento do traumatismo craniano grave ainda não há nenhum estudo em andamento com as estatinas. $^{16}$

\section{Conclusão}

O traumatismo craniano, além de catastrófico, é uma lesão muito heterogêna. Apesar de inúmeros estudos realizados, há várias limitações impostas pela falta de padronização de doses, vias de administração, momento do trauma, desfechos primários e secundários que são observados. A translação dos modelos animais para estudos clínicos de fase III ainda requer um tempo muito prolongado. Em se tratando de uma situação responsável por elevados índices de mortalidade, morbidade e custos para a sociedade, a agilização desse processo torna-se necessário.

\section{Referências}

1 Bales JW, Wagner AK, Kline AE, Dixon CE. Persistent cognitive dysfunction after traumatic brain injury: A dopamine hypothesis. Neurosci Biobehav Rev 2009;33(7):981-1003

2 Gianutsos G, Chute S, Dunn JP. Pharmacological changes in dopaminergic systems induced by long-term administration of amantadine. Eur J Pharmacol 1985;110(3):357-361

3 Dixon CE, Kraus MF, Kline AE, et al. Amantadine improves water maze performance without affecting motor behavior following traumatic brain injury in rats. Restor Neurol Neurosci 1999;14(4): 285-294

4 Meythaler JM, Brunner RC, Johnson A, Novack TA. Amantadine to improve neurorecovery in traumatic brain injury-associated diffuse axonal injury: a pilot double-blind randomized trial. J Head Trauma Rehabil 2002;17(4):300-313

5 Schneider WN, Drew-Cates J, Wong TM, Dombovy ML. Cognitive and behavioural efficacy of amantadine in acute traumatic brain injury: an initial double-blind placebo-controlled study. Brain Inj 1999;13(11):863-872

6 Whyte J, Katz D, Long D, et al. Predictors of outcome in prolonged posttraumatic disorders of consciousness and assessment of medication effects: A multicenter study. Arch Phys Med Rehabil 2005;86(3):453-462

7 Ballesteros J, Güemes I, Ibarra N, Quemada JI. The effectiveness of donepezil for cognitive rehabilitation after traumatic brain injury: a systematic review. J Head Trauma Rehabil 2008;23(3): 171-180

8 Blount PJ, Nguyen CD, McDeavitt JT. Clinical use of cholinomimetic agents: a review. J Head Trauma Rehabil 2002;17(4): 314-321

9 Bourgeois JA, Bahadur N, Minjares S. Donepezil for cognitive deficits following traumatic brain injury: a case report. J Neuropsychiatry Clin Neurosci 2002;14(4):463-464

10 Foster M, Spiegel DR. Use of donepezil in the treatment of cognitive impairments of moderate traumatic brain injury. J Neuropsychiatry Clin Neurosci 2008;20(1):106

11 Fujiki M, KuboT, Kamida T, et al. Neuroprotective and antiamnesic effect of donepezil, a nicotinic acetylcholine-receptor activator, on rats with concussive mild traumatic brain injury. J Clin Neurosci 2008;15(7):791-796
12 Fujiki M, Hikawa T, Abe T, Ishii K, Kobayashi H. Reduced short latency afferent inhibition in diffuse axonal injury patients with memory impairment. Neurosci Lett 2006;405(3): 226-230

13 Chen Y, Shohami E, Constantini S, Weinstock M. Rivastigmine, a brain-selective acetylcholinesterase inhibitor, ameliorates cognitive and motor deficits induced by closed-head injury in the mouse. J Neurotrauma 1998;15(4):231-237

14 Silver JM, Koumaras B, Chen M, et al. Effects of rivastigmine on cognitive function in patients with traumatic brain injury. Neurology 2006;67(5):748-755

15 Silver JM, Koumaras B, Meng X, et al. Long-term effects of rivastigmine capsules in patients with traumatic brain injury. Brain Inj 2009;23(2):123-132

16 Diaz-Arrastia R, Kochanek PM, Bergold P, et al. Pharmacotherapy of traumatic brain injury: state of the science and the road forward: report of the Department of Defense Neurotrauma Pharmacology Workgroup. J Neurotrauma 2014;31(2):135-158

17 Cognitive Remediation after Trauma Exposure: CREATE trial. NCT01416948. Disponível em: https://clinicaltrials.gov/ct2/ show/NCT01416948

18 Zhang HY, Yan H, Tang XC. Non-cholinergic effects of huperzine A: beyond inhibition of acetylcholinesterase. Cell Mol Neurobiol 2008;28(2):173-183

19 Wang Y, Tang XC, Zhang HY. Huperzine A alleviates synaptic deficits and modulates amyloidogenic and nonamyloidogenic pathways in APPswe/PS1dE9 transgenic mice. J Neurosci Res 2012;90(2):508-517

20 Fan Y, Hu J, Li J, et al. Effect of acidic oligosaccharide sugar chain on scopolamine-induced memory impairment in rats and its related mechanisms. Neurosci Lett 2005;374(3):222-226

21 Li J, Wu HM, Zhou RL, Liu GJ, Dong BR. Huperzine A for Alzheimer's disease. Cochrane Database Syst Rev 2008;(2):CD005592

22 Brustovetsky N, Dubinsky JM. Limitations of cyclosporin A inhibition of the permeability transition in CNS mitochondria. J Neurosci 2000;20(22):8229-8237

23 Sharov VG, Todor A, Khanal S, Imai M, Sabbah HN. Cyclosporine A attenuates mitochondrial permeability transition and improves mitochondrial respiratory function in cardiomyocytes isolated from dogs with heart failure. J Mol Cell Cardiol 2007;42(1): $150-158$

24 Alessandri B, Rice AC, Levasseur J, DeFord M, Hamm RJ, Bullock MR. Cyclosporin A improves brain tissue oxygen consumption and learning/memory performance after lateral fluid percussion injury in rats. J Neurotrauma 2002;19(7):829-841

25 Saganová K, Gálik J, Blaško J, Korimová A, Račeková E, Vanický I. Immunosuppressant FK506: focusing on neuroprotective effects following brain and spinal cord injury. Life Sci 2012;91(3-4): $77-82$

26 Setkowicz Z, Guzik R. Injections of vehicle, but not cyclosporin A or tacrolimus (FK506), afford neuroprotection following injury in the developing rat brain. Acta Neurobiol Exp (Warsz) 2007;67(4): 399-409

27 Sullivan PG, Rabchevsky AG, Hicks RR, Gibson TR, Fletcher-Turner A, Scheff SW. Dose-response curve and optimal dosing regimen of cyclosporin A after traumatic brain injury in rats. Neuroscience 2000;101(2):289-295

28 Empey PE, McNamara PJ, Young B, Rosbolt MB, Hatton J. Cyclosporin A disposition following acute traumatic brain injury. J Neurotrauma 2006;23(1):109-116

29 Mazzeo AT, Kunene NK, Gilman CB, Hamm RJ, Hafez N, Bullock MR. Severe human traumatic brain injury, but not cyclosporin a treatment, depresses activated $\mathrm{T}$ lymphocytes early after injury. J Neurotrauma 2006;23(6):962-975

30 Hatton J, Rosbolt B, Empey P, Kryscio R, Young B. Dosing and safety of cyclosporine in patients with severe brain injury. J Neurosurg 2008;109(4):699-707 
31 Aminmansour B, Fard SA, Habibabadi MR, Moein P, Norouzi R, Naderan M. The efficacy of Cyclosporine-A on Diffuse Axonal Injury after Traumatic Brain Injury. Adv Biomed Res 2014;3(3):35

32 Simard JM, Woo SK, Schwartzbauer GT, Gerzanich V. Sulfonylurea receptor 1 in central nervous system injury: a focused review. J Cereb Blood Flow Metab 2012;32(9):1699-1717

33 Patel AD, Gerzanich V, Geng Z, Simard JM. Glibenclamide reduces hippocampal injury and preserves rapid spatial learning in a model of traumatic brain injury. J Neuropathol Exp Neurol 2010;69(12):1177-1190

34 Benvenga S, Campenní A, Ruggeri RM, Trimarchi F. Clinical review 113: Hypopituitarism secondary to head trauma. J Clin Endocrinol Metab 2000;85(4):1353-1361

35 Thum T, Hoeber S, Froese S, et al. Age-dependent impairment of endothelial progenitor cells is corrected by growth-hormonemediated increase of insulin-like growth-factor-1. Circ Res 2007; 100(3):434-443

36 Devesa J, Reimunde P, Devesa P, Barberá M, Arce V. Growth hormone $(\mathrm{GH})$ and brain trauma. Horm Behav 2013;63(2): 331-344

37 Takala J, Ruokonen E, Webster NR, et al. Increased mortality associated with growth hormone treatment in critically ill adults. N Engl J Med 1999;341(11):785-792

38 Yu F, Wang Z, Tchantchou F, Chiu CT, Zhang Y, Chuang DM. Lithium ameliorates neurodegeneration, suppresses neuroinflammation, and improves behavioral performance in a mouse model of traumatic brain injury. J Neurotrauma 2012;29(2):362-374

39 Zhu ZF, Wang QG, Han BJ, William CP. Neuroprotective effect and cognitive outcome of chronic lithium on traumatic brain injury in mice. Brain Res Bull 2010;83(5):272-277

40 Moeller SJ, Honorio J, Tomasi D, et al. Methylphenidate enhances executive function and optimizes prefrontal function in both health and cocaine addiction. Cereb Cortex 2014;24(3):643-653

41 Wagner AK, Drewencki LL, Chen X, et al. Chronic methylphenidate treatment enhances striatal dopamine neurotransmission after experimental traumatic brain injury. J Neurochem 2009;108(4): 986-997

42 Moein H, Khalili HA, Keramatian K. Effect of methylphenidate on ICU and hospital length of stay in patients with severe and moderate traumatic brain injury. Clin Neurol Neurosurg 2006; 108(6):539-542

43 Kim HS, Suh YH. Minocycline and neurodegenerative diseases. Behav Brain Res 2009;196(2):168-179

44 Bye N, Habgood MD, Callaway JK, et al. Transient neuroprotection by minocycline following traumatic brain injury is associated with attenuated microglial activation but no changes in cell apoptosis or neutrophil infiltration. Exp Neurol 2007;204(1): 220-233

45 Kovesdi E, Kamnaksh A, Wingo D, et al. Acute minocycline treatment mitigates the symptoms of mild blast-induced traumatic brain injury. Front Neurol 2012;3:111
46 Dodd S, Dean O, Copolov DL, Malhi GS, Berk M. N-acetylcysteine for antioxidant therapy: pharmacology and clinical utility. Expert Opin Biol Ther 2008;8(12):1955-1962

47 Olive MF, Cleva RM, Kalivas PW, Malcolm RJ. Glutamatergic medications for the treatment of drug and behavioral addictions. Pharmacol Biochem Behav 2012;100(4):801-810

$48 \mathrm{Yi} \mathrm{JH}$, Hazell AS. N-acetylcysteine attenuates early induction of heme oxygenase- 1 following traumatic brain injury. Brain Res 2005;1033(1):13-19

49 Hoffer ME, Balaban C, Slade MD, Tsao JW, Hoffer B. Amelioration of acute sequelae of blast induced mild traumatic brain injury by $\mathrm{N}$ acetyl cysteine: a double-blind, placebo controlled study. PLoS ONE 2013;8(1):e54163

50 Liu L, Wang J, Zhao L, et al. Progesterone increases rat neural progenitor cell cycle gene expression and proliferation via extracellularly regulated kinase and progesterone receptor membrane components 1 and 2. Endocrinology 2009;150(7): 3186-3196

51 Sayeed I, Parvez S, Wali B, Siemen D, Stein DG. Direct inhibition of the mitochondrial permeability transition pore: a possible mechanism for better neuroprotective effects of allopregnanolone over progesterone. Brain Res 2009; 1263:165-173

52 Hua F, Reiss JI, Tang H, et al. Progesterone and low-dose vitamin D hormone treatment enhances sparing of memory following traumatic brain injury. Horm Behav 2012;61(4):642-651

53 Cutler SM, Cekic M, Miller DM, Wali B, VanLandingham JW Stein DG. Progesterone improves acute recovery after traumatic brain injury in the aged rat. J Neurotrauma 2007;24(9): 1475-1486

54 Djebaili M, Hoffman SW, Stein DG. Allopregnanolone and progesterone decrease cell death and cognitive deficits after a contusion of the rat pre-frontal cortex. Neuroscience 2004; 123(2):349-359

55 Béziaud T, Ru Chen X, El Shafey N, et al. Simvastatin in traumatic brain injury: effect on brain edema mechanisms. Crit Care Med 2011;39(10):2300-2307

56 Wang H, Lynch JR, Song P, et al. Simvastatin and atorvastatin improve behavioral outcome, reduce hippocampal degeneration, and improve cerebral blood flow after experimental traumatic brain injury. Exp Neurol 2007;206(1):59-69

57 Lu D, Qu C, Goussev A, et al. Statins increase neurogenesis in the dentate gyrus, reduce delayed neuronal death in the hippocampal CA3 region, and improve spatial learning in rat after traumatic brain injury. J Neurotrauma 2007;24(7): 1132-1146

58 Sierra S, Ramos MC, Molina P, Esteo C, Vázquez JA, Burgos JS. Statins as neuroprotectants: a comparative in vitro study of lipophilicity, blood-brain-barrier penetration, lowering of brain cholesterol, and decrease of neuron cell death. J Alzheimers Dis 2011;23(2):307-318 